\title{
Impact of Marijuana on Response Inhibition: An fMRI Study in Young Adults
}

\author{
Andra M. Smith ${ }^{1}{ }^{*}$, Rocío A. López Zunini ${ }^{1}$, Christopher D. Anderson ${ }^{1}$, Carmelinda A. Longo ${ }^{1}$, \\ Ian Cameron', Matthew J. Hogan', Peter A. Fried ${ }^{3}$ \\ ${ }^{1}$ School of Psychology, University of Ottawa, Ottawa, Canada \\ ${ }^{2}$ The Ottawa Hospital, Department of Diagnostic Imaging, Ottawa, Canada \\ ${ }^{3}$ Department of Psychology, Carleton University, Ottawa, Canada \\ E-mail: "asmith@uottawa.ca \\ Received June 26, 2011; revised July 19, 2011; accepted August 4, 2011
}

\begin{abstract}
Rationale: Marijuana use in adolescence is prevalent and increasing. Understanding the neural correlates of the impact of this use is critical for policy making and for youth awareness. Objectives: The effects of marijuana use on response inhibition were investigated in 19 - 21-year-olds using functional magnetic resonance imaging (fMRI). Methods: Participants were members of the Ottawa Prenatal Prospective Study, a longitudinal study that collected a unique body of information on participants from infancy to young adulthood including: prenatal drug history, detailed cognitive/behavioral performance, and current and past drug use. This information allowed for the control of an unparalleled number of potentially confounding variables including: prenatal marijuana, nicotine, alcohol, and caffeine exposure and offspring alcohol, marijuana, and nicotine use. Ten marijuana users and 14 nonusers that served as controls performed a Go/No-Go task while fMRI blood oxygen level-dependent response was examined. Results: Despite similar task performance, there was a positive relationship between amount of marijuana smoked and activation in right thalamus, premotor cortex and middle frontal gyrus. These regions form part of the neural network responsible for inhibition control. There was also a positive dose dependent relationship with marijuana and activation in inferior parietal lobe and precuneus, also parts of response inhibition pathways. Conclusions: These results suggest a dose dependent alteration in neural functioning during response inhibition after controlling for other prenatal and current drug use. These alterations may be necessary in order to compensate for neural changes in response inhibition circuits caused by long term marijuana use that began during adolescence/young adulthood.
\end{abstract}

Keywords: Prefrontal Cortex, fMRI, Marijuana, Young Adulthood, Response Inhibition

\section{Introduction}

Research has demonstrated that the inability to successfully monitor and inhibit inappropriate behaviours is apparent in substance abusers as well as in other individuals with altered frontal neural circuitry [1]. Such disruption in executive functioning, which can also include selective attention and short term storage of information, initiation of response to relevant information and selfmonitoring of performance in order to achieve a desired goal [1], can cause severe disruption in daily life. Of these elements, however, response inhibition is most vital since it allows for successful adaptation to the environment, recognizing unexpected situations, making plans and changing behaviour accordingly.

Functional magnetic resonance imaging (fMRI) research has shown that response inhibition is mediated by a wide neural network that involves the frontal lobes as well as circuits connecting the frontal lobes with other regions such as the parietal lobes, cerebellum, striatum and thalamus [2-3]. Other observed regions include the premotor area, the supplementary motor area, the dorsolateral and orbitofrontal areas and the anterior cingulate cortex [4].

The 2011 Monitoring the Future Survey reported that there is an increase in American youth marijuana use and 
that there has been an attenuation of perceived risks associated with regular marijuana use [5]. These trends highlight the importance of understanding the impact of marijuana on neural processing.

Using fMRI Tapert et al. [6] compared adolescent marijuana users and nonusers during a Go/No-Go task and found that users showed altered blood oxygen level dependent (BOLD) response during both Go and No-Go trials even after 28 days of abstinence. Users showed greater activation prominently in the dorsolateral prefrontal cortex and the parietal cortex. This was interpreted as an increase in effort required to perform the task. Additionally, using a Stroop test, a measure of response inhibition, and fMRI, [1] compared adult marijuana users and nonusers, with the users testing positive for recent marijuana use in a urine test. Consistent with [6], they found greater activation, in the users compared to nonusers, in the dorsolateral prefrontal cortex. In addition, they also found that users showed decreased activity in the anterior cingulate cortex. These results were interpreted to suggest that the marijuana smokers used different cortical processes than nonusers to perform the task. In two more recent studies, results also illustrated that active marijuana users display greater levels of functional abnormalities than abstinent users in frontal, parietal and cerebellar brain regions as they performed other executive functioning tasks, including visuospatial working memory [7-9]. Again, similar interpretations were suggested, including that marijuana users were required to recruit different neural pathways to perform the tasks and that exposure at a young age may increase the vulnerability to these effects. Despite these findings, further data is needed to more clearly specify and elaborate how early exposure to marijuana affects neural processing in young adulthood. An important requisite in this quest is a well controlled sample.

The differences in neural activation in marijuana users are mostly due to delta-9-tetrahydrocannabinol (THC), marijuana's most active psychoactive ingredient, which acts as a ligand for human cannabinoid receptors. The wide distribution of these receptors in the human brain, with particularly high densities in the cerebellum, parts of the basal ganglia, hippocampus, and many regions of the neocortex, poses great concern for the maintenance of a healthy ability to cognitively process information [3]. Considering the neurodevelopment that occurs during adolescence and young adulthood, specifically, prefrontal cortex development and the subsequent advancement of executive functioning, it is clear that understanding this neural impact of marijuana in youth is imperative. Further understanding this impact was the goal of the present study.

The Ottawa Prenatal Prospective Study (OPPS) is an ongoing longitudinal investigation that was initiated in 1978 , with the primary objective of examining the effects of "soft" prenatal drug exposure on offspring. Children were followed from infancy to young adulthood where detailed information has been collected on their prenatal drug exposure, current and past drug use, cognitive/behavioral performance, and over 4000 lifestyle variables [10-14]. Using this unique sample in combination with the powerful imaging technique, fMRI, and a well established Go/No Go task, the purpose of the present study was to determine if there was a significant relationship between brain activity and marijuana use and if this could be observed in young adults with relatively few years of exposure. Based on previous research where marijuana users and nonusers showed no differences in task performance $[6,8]$, it was hypothesized that there would be no performance differences between groups for the present study. Despite this, marijuana users would require greater activation than controls in brain regions that typically demonstrate response inhibition in order to successfully perform the task, including the prefrontal cortex.

\section{Methods}

\subsection{Participants}

Participants were recruited from the OPPS and signed an informed consent before participation in the study. This study was approved by The Ottawa Hospital ethics board in agreement with the ethical standards laid down in the 1964 Declaration of Helsinki. The sample consisted of ten marijuana users (six males, four females, mean age 20, range of ages 19 - 21) and 14 non-using controls (nine males, five females, mean age of 20, range of ages 19 - 21). Current marijuana use was defined as regular use of marijuana cigarettes per week ( $>1$ joint per week). The users reported smoking an average of 11.48 marijuana joints per week (range of 2 - 37.5 joints per week) on a regular basis and had been smoking marijuana for an average of 4.55 years. The lifetime use for this group would approximate an average of 2697 joints smoked. Previous studies have considered 180 - 1844 lifetime consumption of marijuana as heavy exposure [15]. The nonusers reported never using marijuana regularly. Sporadic marijuana use was reported by three of the 14 controls but no more than one to four times in the past year. No participants had used other illicit drugs on a regular basis or within a month before testing. The illicit drug categories included were amphetamines, crack, cocaine, heroin, mushrooms, hashish, lysergic acid, steroids, solvents, and tranquilizers. Seven of the ten marijuana users smoked nicotine cigarettes on a regular basis while no 
participants from the nonusers control group smoked cigarettes on a regular basis. Cigarette use has been controlled for in the statistical analysis.

Participants from both groups were between the ages of 19 and 21, were right handed, had English as his/her first language and were from middle-class homes. No parents of the participants were reported to have an Axis I diagnosis from the Diagnostic and Statistical Manual of Mental Disorders DSM-IV [16]. Participants completed a comprehensive psychological battery including, the Wechsler Adult Intelligence Scale-III [17], the NEO Personality Inventory [18], and Computerized Diagnostic Interview Schedule for Children (C-DISC) [19], which assessed current psychiatric illness based on DSM-IV criteria. Parents also previously completed the Conners' Parent Rating Scale [20] and provided information on socioeconomic status. No significant differences were found between current marijuana users and nonusers on these scales. Thus, they were not included in the fMRI analyses (see Table 1).

Participants completed a self-report drug questionnaire following the fMRI session. The questionnaire requested information on current and past marijuana use, as well as all other drug use (illicit or non illicit). Participants were asked to abstain from drug use 1 - 2 hours prior to testing.
They were required to provide a urine sample upon arrival at the MRI unit which was sampled for cannabis, amphetamines, opiates, cocaine, creatinine, and cotinine. All metabolite concentrations were adjusted for createnine to control for urine dilution. Significant differences were found between groups for current nicotine and alcohol use and again, have been addressed in the statistical analyses below. Exclusion criteria included (a) diagnosis of DSM-IV Axis I disorder using the C-DISC; (b) positive urine tests for cocaine, opiates, or amphetamines or self-reported regular use of any of these drugs (defined as once/month or more); (c) contraindication to MRI/fMRI (for example a pacemaker, metal implants, accidents leaving metal in eyes, recent surgery, metal dental work (aside from fillings), or insufficient vision for viewing the task); or (d) any abnormalities in structural MRI scans.

Due to previous findings of the impact of prenatal exposure to marijuana [21,22], it was important to consider prenatal exposures. This was one of the benefits of using the OPPS as this information was available for all participants. Detailed information about participant's prenatal drug exposure was previously gathered [10] and these plus the offspring current use details are provided in Table 2 .

Table 1. Environmental and IQ variables for current marijuana users and non using controls.

\begin{tabular}{lccc}
\hline \multicolumn{1}{c}{ Variable } & $\begin{array}{c}\text { Current marijuana users } \\
(n=10, \text { mean(SE) })\end{array}$ & $\begin{array}{c}\text { Nonusers controls } \\
(n=14, \text { mean }(\mathrm{SE}))\end{array}$ & Results (ANOVA) \\
\hline Family income & $31,610(5367.65)$ & $31,611(4707.74)$ & $F(1,21)=0.00(p<0.99)$ \\
WAIS verbal IQ & $106.10(4.10)$ & $116.53(3.60)$ & $F(1,21)=3.66(p<0.07)$ \\
NEO neuroticism & $44.50(15.87)$ & $46.00(8.00)$ & $F(1,18)=0.08(p<0.79)$ \\
NEO extraversion & $49.50(17.85)$ & $59.33(7.44)$ & $F(1,18)=2.94(p<0.10)$ \\
NEO openness & $49.88(10.90)$ & $57.33(11.50)$ & $F(1,18)=2.10(p<0.16)$ \\
NEO agreeableness & $45.88(11.40)$ & $54.75(12.60)$ & $F(1,18)=2.55(p<0.13)$ \\
NEO conscientiousness & $46.75(13.97)$ & $54.92(13.79)$ & $F(1,18)=1.67(p<0.21)$ \\
Connor's (learning problems) & $0.17(2.91)$ & $-0.50(2.42)$ & $F(1,20)=0.36(p<0.55)$ \\
Connor's (anxiety) & $-0.26(0.34)$ & $0.30(1.13)$ & $F(1,20)=1.87(p<0.19)$ \\
\hline
\end{tabular}

No significant differences were observed between the groups for any variable.

Table 2. Drug exposure for marijuana users and nonusers controls.

\begin{tabular}{lccc}
\hline \multicolumn{1}{c}{ Drug exposure } & $\begin{array}{c}\text { Current marijuana users } \\
(n=10, \text { mean(SE) })\end{array}$ & $\begin{array}{c}\text { Nonusers } \\
(n=14, \text { mean }(\mathrm{SE}))\end{array}$ & Results (MANOVA) \\
\hline Prenatal marijuana (joints/week) & $8.82(3.4)$ & $1.12(2.87)$ & $F(1,22)=2.99(p<0.10)$ \\
Prenatal nicotine (cigarettes/day) & $10.41(3.15)$ & $3.09(2.66)$ & $F(1,22)=3.14(p<0.09)$ \\
Current nicotine (cigarettes/day) & $7.75(1.29)$ & $0.00(1.09)$ & $F(1,22)=20.91(p<0.001)$ \\
Current alcohol (drink/week) & $4.77(1.02)$ & $2.00(0.86)$ & $F(1,22)=4.48(p<0.05)$ \\
Prenatal alcohol (AA/day) & $0.13(0.10)$ & $0.28(0.08)$ & $F(1,22)=1.41(p<0.25)$ \\
\hline
\end{tabular}




\subsection{Image Acquisition}

All imaging was performed using a 1.5 Tesla Siemens Magnetom Symphony MR scanner with the quantum gradient set (maximum amplitude $=30 \mathrm{mT} / \mathrm{m}$ and slew rate $=125 \mathrm{~T} / \mathrm{m} / \mathrm{s})$. Subjects lay supine with their head secured in a standard MRI head holder. A conventional T1-weighted spin echo localizer was acquired and used to align the slice orientation for the fMRI scans. This localizer was also used to prescribe a subsequent threedimensional FLASH (TR/TE $11.2 / 21 \mathrm{~ms}$, flip angle $60^{\circ}$, field of view (FOV) $26 \times 26 \mathrm{~cm}^{2}, 256 \times 256$ matrix, slice thickness $1.5 \mathrm{~mm}$ ) volume acquisition used for further structural analyses. Whole brain fMRI was performed using a T2*-weighted echo planar pulse sequence (TR/TE $3,000 / 40 \mathrm{~ms}$, flip angle $90^{\circ}$, FOV $24 \times 24 \mathrm{~cm}^{2}, 64 \times 64$ matrix, slice thickness $5 \mathrm{~mm}, 27$ axial slices, bandwidth $62.5 \mathrm{kHz})$.

\subsection{Procedures}

The cognitive task was presented to the participants on a back projection screen, located at the foot of the patient table, via a mirror attached to the head coil. All lighting in the scanning room was turned off. Button-press responses were recorded via a MRI-compatible fiber optic device (Lightwave Medical, Vancouver, British Columbia, Canada). The stimuli were presented as white letter on a black screen. Participants were asked to press as quickly and accurately as possible and if they made a mistake, to continue without thinking about it. The scanning session began with an initial rest epoch of $9 \mathrm{~s}$ to allow longitudinal magnetic relaxation ( $\mathrm{T} 1$ effects) to stabilize.

\subsection{Go/No-Go Task}

The Go/No-Go blocked design procedure involved presentation of white letters, one at a time, on a black screen for a period of $75 \mathrm{~ms}$, with an inter-stimulus interval of $925 \mathrm{~ms}$. Fifty percent of the stimuli were " $\mathrm{X}$ " and the other $50 \%$ were other capital letters randomly selected from the remainder of the alphabet. $\mathrm{X}$ and non-X stimuli were presented in random order and each epoch was different with respect to order of stimulus presentation. There were two types of conditions. In the "Press for X" condition, participants were instructed to press a button with the right index finger when an $\mathrm{X}$ was presented, and refrain from pressing for all other letters. In the "Press for all letters except X" condition, participants were instructed to refrain from pressing for $\mathrm{X}$ and to press for all other letters with the right index finger. Both conditions were presented in epochs of $27 \mathrm{~s}$ duration, including $3 \mathrm{~s}$ of instructions and 24 stimuli (12 Go and 12 No-Go stimuli). Each Go/No-Go epoch was followed by a $24 \mathrm{~s}$ rest epoch. During instruction epochs, the instruction "Press for X" or "Press for all letters except X" was presented on the screen. During rest epochs, the word REST was presented and the participant was not required to make any motor response. Participants performed a practice session of 10 trials of each Go/No-Go condition outside the scanning room. Within the scanning session, there were four respond to $\mathrm{X}$ and four respond to non- $\mathrm{X}$ epochs, presented in a counterbalanced order, always starting with respond to $\mathrm{X}$.

\subsection{Performance Parameters and Analyses}

Commission errors included any response following a No-Go stimulus (e.g., pressing the button for stimulus B in the 'Press for X' condition and pressing the button for stimulus $\mathrm{X}$ in the "Press for all letters except X" condition) within $900 \mathrm{~ms}$ of stimulus presentation. Omission errors were defined as a failure to respond to a target stimulus within $900 \mathrm{~ms}$. Mean reaction times were calculated for both the "Press for X" and the "Press for all letters except X" conditions for all accurate responses occurring within $900 \mathrm{~ms}$ of stimulus presentation. The behavioral data were analyzed using an ANCOVA with nicotine and alcohol use as covariates. Separate ANCOVAs were performed on the reaction time data, commission errors and omission errors.

Confirming that the non- $\mathrm{X}$ condition involved more response inhibition than the $\mathrm{X}$ condition, the reaction time for the correct responses was longer and the errors of commission were more frequent in the "Press for all letter except X" condition than in the "Press for X" condition. This is consistent with the prediction that withholding responding to a target ( $\mathrm{X}$ in this task) placed greater demand on the neural mechanism for response inhibition. As both conditions entail similar sensory and motor processing, it is then possible to subtract the images for the respond to $\mathrm{X}$ condition from those for the respond to non-X condition to reveal the neural activity related to response inhibition.

\subsection{Image Processing and Analyses}

Prior to statistical analyses, functional images from the first $9 \mathrm{~s}$ of the initial rest block were discarded to ensure that longitudinal magnetic relaxation (T1 effects) had stabilized.

The remaining functional images were realigned to correct for motion by employing the procedures of Friston et al. [23], using Statistical Parametric Mapping (SPM8) software. The motion correction did not exceed 
$1 \mathrm{~mm}$ for any subject. Images were spatially normalized to match the echo planar imaging template provided in SPM8. Following spatial normalization, images were smoothed with an 8-mm full-width at half-maximum Gaussian filter.

\subsection{Imaging Whole Brain Analysis}

All image analyses were performed using SPM8. Individual participant fixed effects analyses were carried out for the comparison of the "Press for all letter except for $\mathrm{X}$ " condition minus the "Press for $\mathrm{X}$ " condition. In addition, individual fixed effect analyses were carried out for the comparison of the "Press for all letters except for X" condition minus the "Rest" condition. One contrast image was created per person for each of the comparisons, and these images were then used for second-level random effects analyses. Group comparisons were performed using both 2 sample t-tests and multiple regressions. Due to the availability of information on each participant's drug use history and exposure, as well as other lifestyle variables, comparisons between the marijuana users and nonusers were performed using several second level analyses, including multiple regression analyses with covariates specified for each.

\section{Results}

\subsection{Drug Questionnaire and Urine Sample Data}

All marijuana users had smoked marijuana during the week of fMRI testing, with four of the ten participants smoking marijuana on the day of testing (two participants smoked one joint in the morning while two smoked throughout the day as was typical for their regular use-but not within 3 hours of the testing session). The average urine cannabis at the time of testing for the group of ten using participants was $460 \mu \mathrm{g} / \mathrm{L}$, with a range from 16 to $1325 \mu \mathrm{g} / \mathrm{L}$ (all $10 \mathrm{had}$ cannabis in their urine). One of the nonusers had smoked one joint 3 days prior to testing and had $45 \mu \mathrm{g} / \mathrm{L}$ in his urine; no other exposure was reported for this participant in the months prior to testing. No other nonuser had cannabis in their urine. The average number of joints smoked by the marijuana users for the 7 days prior to testing was $4.2,4.55$, $3.15,2.75,2.9,4.6$, and 4.35 , and on the day of testing, the average use was 2.5 joints.

The cotinine values for urine samples revealed an average value of $888 \mu \mathrm{g} / \mathrm{L}$ for the marijuana using group (seven of ten were cigarette smokers) while only $9.8 \mu \mathrm{g} / \mathrm{L}$ for the nonusers group (which may be due to second hand smoke exposure as none of the marijuana nonusers smoked cigarettes on a regular basis). Thus, there were significant differences between groups for nicotine use. Therefore, amount of nicotine smoked/day was used as a covariate in whole brain statistical analyses.

The Pearson correlation between the drug questionnaire results and the urine samples for levels of marijuana use was $0.97(p<0.001)$ while that for nicotine (cotinine/creatinine) was $0.91(p<0.001)$. This high concordance validated the use of the self-report drug questionnaire results for current use and drug history.

No participant from either group reported alcohol consumption on the day of imaging. One of the marijuana users reported drinking 15 alcoholic drinks on the day prior to testing but no other participant reported more than seven drinks for the 2 days prior to testing. This reduces the possibility that the results were related to the acute effects of alcohol consumption, given its short half life.

\subsection{Performance Data}

There were no significant performance differences between marijuana users and nonusers on reaction time, commission errors and omission errors while controlling for nicotine and alcohol use (Table 3).

\subsection{Whole Brain fMRI Analyses}

Fixed effects analyses revealed an expected pattern of activation from the non marijuana using participants.

Table 3. Performance data for the two conditions of the Go/No-Go task for marijuana users and nonusers.

\begin{tabular}{lccc}
\hline \multicolumn{1}{c}{ Performance measure } & $\begin{array}{c}\text { Marijuana users } \\
(n=10, \text { mean(SE) })\end{array}$ & $\begin{array}{c}\text { Nonusers } \\
(n=14, \text { mean(SE)) }\end{array}$ & Results (ANCOVA) \\
\hline Errors of omission (Press for X) & $0.20(0.13)$ & $0.14(0.14)$ & $F(1,19)=0.63(p<0.44)$ \\
Errors of omission (Press for all except X) & $0.40(0.22)$ & $0.29(0.22)$ & $F(1,19)=0.21(p<0.65)$ \\
Errors of commission (Press for X) & $1.00(0.30)$ & $0.57(0.17)$ & $F(1,19)=1.40(p<0.25)$ \\
Errors of commission (Press for all except X) & $4.10(1.00)$ & $4.57(1.20)$ & $F(1,19)=1.34(p<0.26)$ \\
Reaction time (s, Press for X) & $0.40(0.02)$ & $0.39(0.02)$ & $F(1,19)=0.23(p<0.63)$ \\
Reaction time (s, Press for all except X) & $0.41(0.01)$ & $0.41(0.02)$ & $F(1,19)=0.01(p<0.91)$ \\
\hline
\end{tabular}


This was used as a confirmation that the task was recruiting the response inhibition circuitry as anticipated. Although the ideal contrast was "non- $X$ " minus " $X$ ", the power, when considering covariates, was too small to report significant differences between conditions. However, the "Press for all letters except X" minus rest contrast had sufficient power to reveal the expected pattern of activation. This is presented in Figure 1 for the nonusers and areas included the dorsolateral prefrontal cortex, premotor cortex, supplementary motor cortex, cerebellum, insula and superior temporal gyrus.

Random effect analyses between groups were performed using the "Press for all letters except X" condition minus the "Press for X" condition. A 2 sample t-test analysis without any covariates was used to confirm that there were differences between users and nonusers during challenge of the response inhibition circuitry. There were no areas that showed significantly less activation in marijuana users than nonusers. However, marijuana users did show significantly more activation than nonusers in typical response inhibition areas, including the precentral gyrus, superior, middle, orbital and inferior frontal gyri, lingual gyrus and supramarginal gyrus. Again, however, the power was insufficient when controlling for other drug exposures. For example prenatal marijuana has been shown to play a role in response inhibition [21], and even though there was no significant difference between groups for prenatal drug effects, it was deemed important to determine if in fact these exposures were impacting the results of current marijuana use on neural functioning.

Thus, two multiple regression analyses were performed, with this contrast of "Press for all letters except $\mathrm{X}$ " minus "Press for $\mathrm{X}$ ", using prenatal marijuana exposure and prenatal nicotine exposure as covariates. The results suggest that these did not contribute to the differences between groups. In addition, further multiple regressions of the same contrast were performed with: 1) current alcohol as a covariate; 2) current nicotine as a covariate; 3) current alcohol and current nicotine together as covariates; and 4) current alcohol, current nicotine and prenatal marijuana together as covariates. The results suggest that these other variables do contribute to the differences between groups as the results were no longer significant with these analyses. Therefore, to ensure sufficient power while still controlling for other drug exposures, further analyses were conducted using the "Press for all letters except for X" condition minus the "Rest" condition. It was anticipated that this type of analysis would allow for the identification of differences between groups in motor, visual and other brain regions that otherwise would not be possible to identify using the "Press for all letters except X" condition minus the "Press

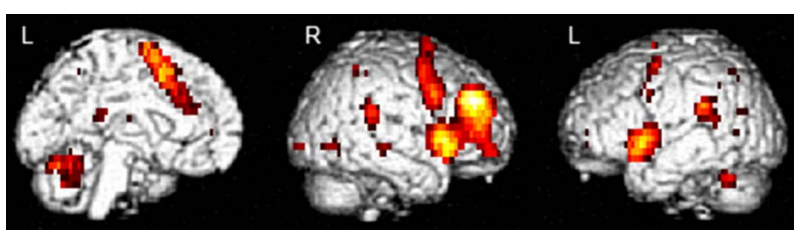

Figure 1. Non marijuana smoking group analysis rendered at $\mathrm{FWE}=\mathbf{0 . 0 5}$, corrected for multiple comparisons, for clusters larger than 50 voxels. $L$ represents the view from the left side of the brain while $R$ represents the view from the right side of the brain. The left most image is a medial sagittal view of the cerebellar and premotor/supplementary motor activations for the "Press for all letters except $X$ " minus "Rest" contrast. The middle and right most image represent lateral sagittal views of the prefrontal cortex and parietal area activations for the same contrast.

\section{for X" condition.}

Multiple regression analyses of the new contrast yielded a significant positive relationship between amount of marijuana use and neural activity even with each of the prenatal and current drug variables as covariates. In addition, the results were also significant when controlling for acute marijuana use (by removal of those participants that smoked marijuana on the day of testing). Only the results from the analysis with prenatal marijuana, current nicotine and current alcohol together as covariates are reported below.

The most robust effect of this study was the significant increase in neural activation in several regions as the amount of "self reported" marijuana smoked increased. These results were observed for the 'Press for all letters except $\mathrm{X}$ ' minus 'Rest' contrast, at a $p$ value corrected multiple comparisons for cluster level at 0.05 , in a large cluster of 2578 voxels that included the right thalamus $(x$ $y z=3-18$ 10; Figure 2), the right premotor cortex ( $x$ y z $=336$ 30; Figure 2) and the right middle frontal gyrus $(x$ y $z=3318$ 60; Figure 2). Results also showed greater activation in the inferior parietal lobe/supramarginal gyrus ( $x$ y $z=48-4850$ ) and the precuneus ( $x$ y $z=3$ $-6660)$, uncorrected at 0.05 ; cluster size of 689 voxels as marijuana use increased.

\section{Discussion}

This study examined BOLD fMRI response among regular current marijuana users and nonusers during a Go/No-Go task. Although differences in behavioral performance were non-significant, the two groups differed in their pattern of neural activation, with more BOLD activity occurring in a dose dependent manner as the quantity of marijuana use increased. Furthermore, the increased activity was still significant after controlling for other drugs such as alcohol, nicotine and prenatal marijuana. 


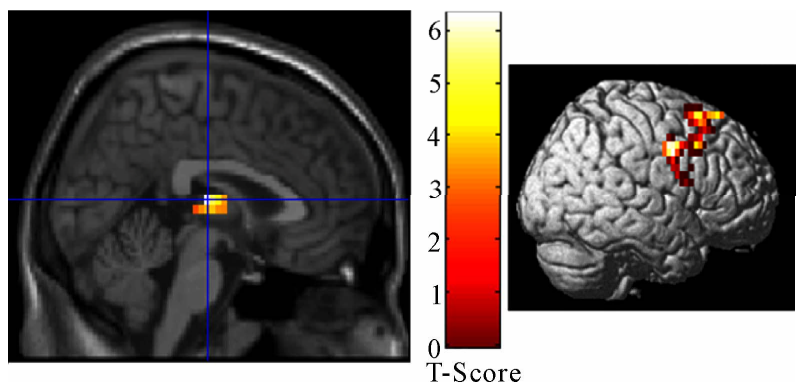

Figure 2. Images representing the positive relationship between marijuana use and thalamic activation (left most image) and the right prefrontal cortex activation (right most image) for the "Press for All Letters except $X$ " minus "Rest" contrast at FWE, $p=0.05$, corrected for multiple comparisons, with only clusters with more than 200 significantly activated voxels.

The most substantial differences in activation were found to be right lateralized in the premotor cortex and the middle frontal gyrus or dorsolateral prefrontal cortex. Response inhibition in healthy controls involves a distributed network that includes these areas as well as parietal areas $[3,24,25]$. During response inhibition, the premotor cortex is involved in response competition and the preparatory process leading to correct initiation or suppression of movement [24,26]. Given that there were non-significant behavioral differences in errors of commission between the two groups it is unlikely that this increased activation of the premotor cortex is related to increased motor responses in the users. Also, [4] found that mostly left premotor cortex is involved during preparation to respond. Thus, the findings from the present study suggest that marijuana smokers may need to compensate by recruiting homologous contralateral areas in order to correctly initiate or suppress responses.

In a response inhibition study by Casey et al. [27], it was found that the volume of activation in the middle frontal gyrus is correlated with age, suggesting that this structure may be important in the developmental improvement of inhibitory abilities. Furthermore, several studies support that the development of the response inhibition circuitry continues to develop well into late adolescence [28-32]. Given that the OPPS sample is comprised of young adults who on average have been smoking marijuana for 4.5 years (i.e. started smoking during adolescence), it is possible that their exposure to marijuana over those years may have compromised middle frontal gyrus development and thus, explain the dose dependent increase in activation. These results emphasize the importance of early education about the potential cognitive impact of early exposure to marijuana.

Another brain region that showed a significant positive relationship between activation and amount of marijuana smoked was the right thalamus. The thalamus is included in various circuits including the frontal-striatal-thalamic and the cingulo-opercular networks [2]. The frontal-striatal-thalamic circuit has been found to support the development of inhibitory control and correlate with better performance on inhibitory tasks [30,31]. The cinguloopercular network, which also includes the thalamus, among other brain regions, is thought to support response rate. Response rate refers to the ability to apply cognitive skills in a consistent and flexible manner depending on a task's demands [2]. A voxel-based morphometry study revealed that marijuana users had increased gray matter density in the right precentral gyrus and right thalamus compared to nonusers [33]. The authors speculated that changes in one component of the brain, gray matter in this case, may be compensated for by changes in a neighbouring component, for example, gray matter displacement caused by a decrease of nearby white matter. Therefore, it is possible that the greater activation found in the right thalamus of marijuana users in the present study may be related to a change in white matter in other areas forming part of the inhibitory pathways. Consequently, compensation on such circuits may have occurred by increased activation of the right thalamus in order for users to keep up with the behavioral demands of the "Press for all except X" condition. The inability to assess white and gray matter volumes in the present study is a limitation and future research is required.

The present study also revealed a trend for a positive relationship between amount of marijuana smoked and greater bilateral activation in the inferior parietal lobe. Several imaging studies have also found increased parietal lobe activation in marijuana users [1,6,9]. More specifically, right parietal regions have been implicated in sustained attention [34], with neuroimaging studies reporting parietal activation during attentionally demanding tasks to be in the superior, rather than inferior, parietal lobule [3]. Together, these results suggest that marijuana users may recruit additional parietal regions in order to properly sustain their attention during response inhibition tasks.

Additionally, the parietal lobes are also part of the frontal-parietal circuit, which has also been associated with inhibitory control and working memory [35]. This network has been found to continue to reorganize through adolescence, becoming more distinct and segregated from one another and further integrating long distance connections [36]. As previously mentioned, the OPPS sample of users had been smoking marijuana since adolescence. Therefore, it is possible that the relationship between neural activation and marijuana consumption may be due to either a delay or to an altered development of such a network due to marijuana exposure during years where response inhibition circuits are still under 
development.

Another positively related trend from the present study was observed in the precuneus. This region is thought to have a role in error awareness and monitoring [24,37], which is an important aspect of prefrontal cortex function since error detection allows for the correction and improvement of task performance [38]. Support for this evidence is found in electrophysiological and lesion studies that propose that this region may be involved in evaluative functions such as monitoring behaviour [37, 39]. Moreover, Nagahama et al. [40] have found that the precuneus is activated when external feedback shifts from "correct" to "incorrect" during tasks where subjects are required to alter stimulus-response judgments. Although during the Go/No-Go task, in the present study did not introduce an error awareness task, these findings may indicate that marijuana users need to work harder in order to monitor their response and be aware of errors during their performance. However, further testing with a response inhibition task that includes error awareness recognition should be carried out in order to confirm this result.

Although other fMRI studies researching response inhibition in marijuana users have found greater activation in prefrontal regions including the dorsolateral prefrontal cortex $[1,6]$, the present study provides further evidence that other brain regions of response inhibition circuitry may also be altered. Other frontal areas and regions of the parietal lobe, as well as subcortical areas are also affected by marijuana exposure. Moreover, the three circuits involved in response inhibition, namely the frontal-striatal thalamic circuit, the cingulo-opercular circuit and frontal-parietal circuit, are all still under development during adolescence [27,30-32,41,42]. Therefore, the overactive brain regions observed in this investigation may be due not only to the current marijuana use but also to the relatively long term marijuana exposure during those crucial years in adolescence.

The strength of this study was the ability to control for an unparalleled number of lifestyle variables including IQ, current nicotine and alcohol use and prenatal marijuana, nicotine, and alcohol exposure. The well controlled sample strengthens the validity of the results and provides outcomes that are able to shed light on more exclusive contributions of marijuana on the response inhibition network than previous studies.

The limitations of the study include that the sample was small and a primarily Caucasian, middle-class population. Thus, these results cannot be generalized to other ethnic or socioeconomic status populations. However, this is a low risk population and these effects are significant, suggesting that a high risk population would be even more likely to show a negative impact of mari- juana use given the other risk factors.

The present study also used a block design rather than an event related design. A block design does not permit the separation of Go from the No-Go components of the brain activity. Thus, an event-related study may have helped to separate response inhibition from other cognitive processes. It is also difficult to ever truly remove a drug's effect from a BOLD study and thus it must be considered that current use of nicotine impacted the results even after using it as a covariate. Similarly, there was no measure of alcohol consumption on the day of testing other than the self-report of each participant. Although the self-report and urine sample values were highly correlated for those drugs tested in the urine, this was an oversight for the alcohol consumption and should be rectified with the addition of a breath alcohol level assessment in future research. Finally, there was no abstinence period for the participants of either group. However, careful statistical analyses were performed including and not including those participants who smoked marijuana on the day of testing. Even though these analyses had less power than the reported results, the same positive relationship between amount of marijuana smoked and neural activity was observed. This suggests that the reported results are indicative of the regular marijuana use and not only acute marijuana effects. Future research will test participants who have stopped using marijuana for at least 6 months.

In conclusion, adolescent use of marijuana can have detrimental effects on the brain that can be observed in young adulthood. The findings in this study suggest that increase in neural activation with increased marijuana use may be due to a form of neural compensation or an altered neural development, or both. Also, this may occur not only in the prefrontal cortex but also in the extensive neural network required for inhibitory control, a cognitive process important for executive functioning and thus success in establishing and reaching appropriate goals during adulthood.

\section{Acknowledgments}

The research was funded through an Ontario Research and Development Challenge Fund grant. The authors would like to acknowledge the contributions of Robert Gray and the MRI technologists at the Ottawa Hospital.

\section{References}

[1] S. A. Gruber and D. A. Yurgelun-Todd, "Neuroimaging of Marijuana Smokers during Inhibitory Processing: A Pilot Investigation," Cognitive Brain Research, Vol. 23, No. 1, 2005, pp. 107-118. doi:10.1016/i.cogbrainres.2005.02.016 
[2] B. Luna, A. Padmanabhan and K. O'Hearn, "What Has fMRI Told Us about the Development of Cognitive Control through Adolescence?" Brain and Cognition, Vol. 72, No. 1, 2010, pp. 101-113. doi:10.1016/j.bandc.2009.08.005

[3] H. Garavan, T. J. Ross and E. A. Stein, "Right Hemispheric Dominance of Inhibitory Control: An Event-Related Functional MRI Study," Proceedings of the National Academy of Science, Vol. 96, No. 14, 1999, pp. 8301-8306. doi:10.1073/pnas.96.14.8301

[4] J. Watanabe, M. Sugiura, I. K. Sato, Y. Sato, M. Yasuhiro, M. Yoshihiko, H. Fukuda and R. Kawashima, "The Human Prefrontal and Parietal Association Cortices Are Involved in NO-GO Performances: An Event Related fMRI Study," Neuroimage, Vol. 17, No. 3, 2002, pp. 12071216. doi:10.1006/nimg.2002.1198

[5] L. D. Johnston, P. M. O’Malley, J. G. Bachman and J. E. Schulenberg, "Monitoring the Future National Results on Adolescent Drug Use: Overview of Key Findings, 2010," Institute for Social Research, The University of Michigan, Ann Arbor, 2011.

[6] S. F. Tapert, A. D. Schweinsburg, S. P. Drummond, M. P. Paulus, S. A. Brown, T. T. Yang and L. R. Frank, "Functional MRI of Inhibitory Processing in Abstinent Adolescent Marijuana Users," Psychopharmacology, Vol. 194, No. 2, 2007, pp. 173-183. doi:10.1007/s00213-007-0823-y

[7] D. A. Schweinsberg, B. C. Schweinsberg, K. L. Medina, T. McQueeny, S. A. Brown and S. F. Tapert, "The Influence of Recency of Use on fMRI Response during Spatial Working Memory in Adolescent Marijuana Users," Journal of Psychoactive Drugs, Vol. 42, No. 3, 2010, pp. 401-412.

[8] A. M. Smith, C. Longo, P. A. Fried, M. J. Hogan and I. Cameron, "Effects of Marijuana on Visuospatial Working Memory: An fMRI Study in Young Adults," Psychopharmacology, Vol. 210, No. 3, 2010, pp. 429-438. doi:10.1007/s00213-010-1841-8

[9] A. D. Schweinsburg, S. A. Brown and S. F. Tapert, "The Influence of Marijuana Use on Neurocognitive Functioning in Adolescents," Current Drug Abuse Reviews, Vol. 1, No. 1, 2008, pp. 99-111.

[10] P. A. Fried, B. Watkinson, A. Grant and R. K. Knights, "Changing Patterns of Soft Drug Use Prior to and during Pregnancy: A Prospective Study," Drug and Alcohol Dependence, Vol. 6 , No. 5,1980 , pp. 323-343. doi:10.1016/0376-8716(80)90199-4

[11] P. A. Fried, B. Watkinson and R. Gray, "Differential Effects on Cognitive Functioning in 9-to 12-Year Olds Prenatally Exposed to Cigarettes and Marihuana," Neurotoxicology and Teratology, Vol. 20, No. 3, 1998, pp. 293-306. doi:10.1016/S0892-0362(97)00091-3

[12] P. A. Fried, "The Ottawa Prenatal Prospective Study (OPPS): Methodological Issues and Findings-It's Easy to Throw the Baby Out with the Bath Water," Life Sciences, Vol. 56, No. 23-24, 1995, pp. 2159-2168. doi:10.1016/0024-3205(95)00203-I

[13] P. A. Fried and B. Watkinson, "36 - 48-Month Neurobe- havioral Follow-up of Children Prenatally Exposed to Marijuana, Cigarettes and Alcohol," Developmental and Behavioral Pediatrics, Vol. 11, No. 2, 1990, pp. 49-58. doi:10.1097/00004703-199004000-00003

[14] P. A. Fried and B. Watkinson, "Differential Effects on Facets of Attention in Adolescents Prenatally Exposed to Cigarettes and Marihuana," Neurotoxicology and Teratology, Vol. 23, No. 5, 2001, pp. 421-430. doi:10.1016/S0892-0362(01)00160-X

[15] S. Bava, L. R. Frank, T. McQueeny, B. C. Schweinsburg, A. D. Schweinsburg and S. F. Tapert, "Altered White Matter Microstructure in Adolescent Substance Users," Psychiatry Research: Neuroimaging, Vol. 173, No. 3, 2009, pp. 228-237. doi:10.1016/j.pscychresns.2009.04.005

[16] American Psychiatric Association, "Diagnostic and Statistical Manual of Mental Disorders-Fourth Edition (DSMIV)," American Psychiatric Association, Washington, 1994.

[17] D. Wechsler, "Wechsler Adult Intelligence Scale," 3rd Edition, The Psychology Corporation, San Antonio, 1997.

[18] P. T. Costa Jr. and R. R. McCrae, "NEO PI Professional Manual," Psychological Assessment Resources, Odessa, 1989.

[19] W. Bacon, "NIMH-Computerized Diagnostic Interview Schedule for Children-Version IV (C-DISC 4)," Columbia University, New York, 1997.

[20] C. H. Goyette, C. K. Conners and R. F. Ulrich, "Normative Data on Revised Conners' Parent and Teacher Rating Scales," Journal of Abnormal Child Psychology, Vol. 6, No. 2, 1978, pp. 221-236. doi:10.1007/BF00919127

[21] A. M. Smith, P. A. Fried, M. J. Hogan and I. Cameron, "Effects of Prenatal Marijuana on Response Inhibition: An fMRI Study in Young Adults," Neurotoxicology and Teratology, Vol. 26, No. 4, 2004, pp. 533-542. doi:10.1016/j.ntt.2004.04.004

[22] G. A. Richardson, C. Ryan, J. Willford, N. L. Day and L. Goldschmidt, "Prenatal Alcohol and Marijuana Exposure: Effects on Neuropsychological Outcomes at 10 Years," Neurotoxicology and Teratology, Vol. 24, No. 3, 2002, pp. 309-320. doi:10.1016/S0892-0362(02)00193-9

[23] K. J. Friston, J. Ashburner, J. B. Poline, C. D. Frith, J. D. Heather and R. S. J. Frackowiak, "Spatial Realignment and Normalization of Images," Human Brain Mapping, Vol. 3, No. 3, 1995, pp. 165-189. doi:10.1002/hbm.460030303

[24] V. Menon, N. E. Adleman, C. D. White, G. H. Glover and A. L. Reiss, "Error-Related Brain Activation during a Go/NoGo Response Inhibition Task," Human Brain Mapping, Vol. 12, No. 3, 2001, pp. 131-143. doi:10.1002/1097-0193(200103)12:3<131::AID-HBM10 10>3.0.CO;2-C

[25] S. Yamaguchi, D. Zheng, T. Oka and H. Bokura, "The Key Locus of Common Response Inhibition Network for No-Go and Stop Signals," Journal of Cognitive Neuroscience, Vol. 20, No. 8, 2008, pp. 1434-1442. doi:10.1162/jocn.2008.20100 
[26] J. R. Booth, D. D. Burman, J. R. Meyer, Z. Lei, B. L. Trommer, N. D. Davenport, W. Li, T. B. Parrish, D. R. Gitelman and M. M. Mesulam, "Neural Development of Selective Attention and Response Inhibition," Neuroimage, Vol. 20, No. 2, 2003, pp. 737-751. doi:10.1016/S1053-8119(03)00404-X

[27] B. J. Casey, R. J. Trainor, J. L. Orendi, A. B. Schubert, L. E. Nystrom, J. N. Giedd, F. X. Astellanos, J. V. Haxby, D. C. Noll and J. D. Cohen, "A Developmental Functional MRI Study of Prefrontal Activation during Performance of a Go-No-Go Task," Journal of Cognitive Neuroscience, Vol. 9, No. 6, 1997, pp. 835-847. doi:10.1162/jocn.1997.9.6.835

[28] C. F. Geier, R. Terwilliger, T. Teslovich, K. Velanova and B. Luna, "Immaturities in Reward Processing and Its Influence on Inhibitory Control in Adolescence," Cerebral Cortex, Vol. 20, No. 7, 2010, pp. 1613-1629. doi:10.1093/cercor/bhp225

[29] S. Durston, M. C. Davidson, N. Tottenham, A. Galvan, J. Spicer, J. A. Fossella and B. J. Casey, "A Shift from Diffuse to Focal Cortical Activity with Development," Developmental Science, Vol. 9, No. 1, 2006, pp. 1-8. doi:10.1111/j.1467-7687.2005.00454.x

[30] K. Rubia, A. B. Smith, E. Taylor and M. Brammer, "Linear Age-Correlated Functional Development of Right Inferior Fronto-Striato-Cerebellar Networks during Response Inhibition and Anterior Cingulate during ErrorRelated Processes," Human Brain Mapping, Vol. 28, No. 11, 2007, pp. 1163-1177. doi:10.1002/hbm.20347

[31] K. Rubia, A. B. Smith, J. Woolley, C. Nosarti, I. Heyman, E. Taylor and M. Brammer, "Progressive Increase of Frontostriatal Brain Activation from Childhood to Adulthood during Event-Related Tasks of Cognitive Control," $\mathrm{Hu}$ man Brain Mapping, Vol. 27, No. 12, 2006, pp. 973-993. doi:10.1002/hbm.20237

[32] K. Velanova, M. E. Wheeler and B. Luna, "Maturational Changes in Anterior Cingulate and Frontoparietal Recruitment Support the Development of Error Processing and Inhibitory ContRol," Cerebral Cortex, Vol. 18, No. 11, 2008, pp. 2505-2522. doi:10.1093/cercor/bhn012

[33] J. A. Matochik, D. A. Eldreth, J. L. Cadet and K. I. Bolla, "Altered Brain Tissue Composition in Heavy Marijuana Users," Drug and Alcohol Dependence, Vol. 77, No. 1, 2005, pp. 23-30. doi:10.1016/j.drugalcdep.2004.06.011

[34] S. P. A. Drummond, A. Bischoff-Grethe, D. F. Dinges, L. Ayalon, C. M. Mednick and M. J. Meloy, "The Neural
Basis of the Psychomotor Vigilance Task," Sleep, Vol. 28, No. 9, 2005, pp. 1059-1068.

[35] N. U. Dosenbach, K. M. Visscher, E. D. Palmer, F. M. Miezin, K. K. Wenger and H. C. Kang, "A Core System for the Implementation of Task Sets," Neuron, Vol. 50, No. 5, 2006, pp. 799-812. doi:10.1016/j.neuron.2006.04.031

[36] D. A. Fair, N. U. Dosenbach, J. A. Church, A. L. Cohen, S. Brahmbhatt and F. M. Miezin, "Development of Distinct Control Networks through Segregation and Integration," Proceedings of the National Academy of Science USA, Vol. 104, No. 33, 2007, pp. 13507-13512. doi:10.1073/pnas.0705843104

[37] R. Hester, L. Nestor and H. Garavan, "Impaired Error Awareness and Anterior Cingulate Cortex Hypoactivity in Chronic Cannabis Users," Neuropsychopharmacology, Vol. 34, No. 11, 2009, pp. 2450-2458. doi:10.1038/npp.2009.67

[38] R. I. Block, D. S. O’Leary, J. C. Ehrhardt, J. C. Augustinack, M. M. Ghoneim and S. Arndt, "Effects of Frequent Marijuana Use on Brain Tissue Volume and Composition," Neuroreport, Vol. 11, No. 3, 2000, pp. 491-496. doi:10.1097/00001756-200002280-00013

[39] B. A. Vogt, D. M. Finch and C. R. Olson, "Functional Heterogeneity in Cingulate Cortex: The Anterior Executive and Posterior Evaluative Regions," Cerebral Cortex, Vol. 2, No. 6, 1992, pp. 435-443. doi:10.1093/cercor/2.6.435-a

[40] Y. Nagahama, T. Okada, Y. Katsumi, T. Hayashi, H. Yamauchi, N. Sawamoto, K. Toma, K. Nakamura, T. Hanakawa, J. Konishi, H. Fukuyama and H. Shibasaki, "Transient Neural Activity in the Medial Superior Frontal Gyrus and Precuneus Time Locked with Attention Shift Between Object Features," Neuroimage, Vol. 10, No. 2, 1999, pp. 193-199. doi:10.1006/nimg.1999.0451

[41] B. Luna and J. A. Sweeney, "Studies of Brain and Cognitive Maturation through Childhood and Adolescence: A Strategy for Testing Neurodevelopmental Hypotheses," Schizophrenia Bulletin, Vol. 27, No. 3, 2001, pp. 443455.

[42] K. Rubia, S. Overmeyer, E. Taylor, M. Brammer, S. C. Williams and A. Simmons, "Functional Frontalisation with Age: Mapping Neurodevelopmental Trajectories with fMRI," Neuroscience and Biobehavioral Reviews, Vol. 24, No. 1, 2000, pp. 13-19. doi:10.1016/S0149-7634(99)00055-X 